role of genuine community participation based on both scientific evidence and international human rights principles.

\section{Symposium Presentations}

\section{S01 - A strategy for HIV/STI prevention in low and middle income countries}

\section{S01.1 HIV AND STI PREVENTION: WHAT IS AN INTERVENTION?}

James Hargreaves* . London School of Hygiene \& Tropical Medicine, London, UK

10.1136/sextrans-2015-052270.17

Structural intervention approaches for HIV prevention work to remove social barriers to the use of HIV prevention services and promote the adoption of behaviours that reduce the risk of HIV transmission. These approaches act at the health policy level to support the delivery of HIV prevention tools; at the health systems level to support the integration of HIV prevention with other health services; and at the community level to promote of critical enabler interventions, such as peer-based community empowerment programmes developed through safe spaces often physically within health facilities. They also take the form of policy integration and resources with non-health sector structures that reach populations at risk in large numbers, such as in social development, education, microfinance/poverty alleviation. While there have been some evidence syntheses on the effectiveness of specific types of structural interventions, there is no review or summary of the evidence on the effectiveness of such interventions as a whole. This paper will provide a synthesis of the evidence on the effectiveness of structural interventions for HIV prevention, by providing a review of reviews of the literature with a discussion of the strength of the evidence from reviews and primary studies. The paper will summarise the key findings from these reviews with an aim to provide recommendations for the use of structural interventions for HIV prevention.

\section{S01.2 SYSTEMATIC REVIEWS OF THE EFFICACY AND EFFECTIVENESS OF BIOLOGICAL, BEHAVIOURAL AND STRUCTURAL HIV PREVENTION INTERVENTIONS}

Shari Krishnaratne* . London School of Hygiene \& Tropical Medicine, London, UK

10.1136/sextrans-2015-052270.18

Behavioural interventions for HIV prevention work by encouraging people to change behaviours that may make them more susceptible to HIV infection, or more likely to infect another person. There is some evidence that such interventions have led to reductions in HIV risk behaviours, such as having multiple partners, and improvements in other outcomes, such as increased condom use, increased testing, and improved treatment adherence. Behaviour is influenced heavily by socio-cultural contexts and as such, behavioural interventions must be sensitive to these contexts in order to be effective. Examples of behavioural interventions include individual and group level counselling, and providing information and guidelines through community outreach and mass media campaigns.

This paper will provide a synthesis of the evidence on the effectiveness of behavioural interventions for HIV prevention. A review of reviews methodology will be followed to identify relevant primary studies. The strength of the evidence from these reviews will be evaluated and recommendations will be made.

\section{SO1.3 DELIVERY OF HIV/STI PREVENTION INTERVENTIONS: PREVENTION CASCADES}

Geoff Garnett* . Bill and Melinda Gates Foundation, Seattle, USA

10.1136/sextrans-2015-052270.19

The HIV treatment cascade has been a powerful illustrative tool used to explore the performance of HIV treatment programs. The cascade steps through the recruitment of HIV-infected individuals into treatment programs and the effectiveness of those programs, and despite its conceptual flaws provides an intuitively appealing snapshot of performance. Because of its advocacy potential attempts have been made to generate a prevention cascade, covering those HIV positive and negative or to integrate prevention into the treatment cascade generating a 'prevention, treatment and care cascade'. However, these tend to diminish the focus on prevention, confuse different interventions and do not provide a simple scheme through which to measure performance. Here we explore the required elements for prevention cascades for HIV and other sexually transmitted infections and identify the steps linking the delivery of interventions with their use in populations. Starting from the susceptible population at risk we can consider whether the intervention is available to them, whether they uptake the intervention, whether they adhere to the intervention and what the efficacy of the intervention is. Cascades for the delivery, use and effectiveness of prevention products should be able to identify who has been protected, what the key failures in protection are and the relative importance of system and product characteristics. In explaining theoretically how we might think about prevention programs we hope that empirical studies will consider adopting this framework, which has guided some thinking about voluntary medical male circumcision programs and HIV pre-exposure prophylaxis programs. In HIV treatment global targets and indicators have been set based on the treatment cascade and without similar targets for other prevention interventions they are likely to be neglected.

\section{S01.4 MODELLING THE COST-EFFECTIVENESS OF HIV PREVENTION INTERVENTIONS}

Tim Hallett*. Imperial College London, London, UK

\subsection{6/sextrans-2015-052270.20}

Ambitious targets have been set for HIV prevention. We will begin by examining assumptions for HIV prevention scale-up in the light of the concept of the 'prevention cascade' using available data. Then, we will show that a key part of optimising prevention impact will be in allocating available funding according to four main factors - intervention, population, geography and time. The impact of flexibility or constraint on each of these will be illustrated, providing recommendations for how international and domestic decisions can be taken to maximise epidemic impact. Finally, we will examine methods to evaluate the evidence of new prevention technologies to determine the scale of investment they might optimally attract. We will show that this requires a holistic view of the range of tools available in 